\title{
Multi-stakeholders Comparative Assessment of Freeway Traffic Conditions by Data Envelopment Analysis
}

\author{
Shen Zhang ${ }^{1, ~ a ~}$ and Wei Quan ${ }^{1, b}$ \\ ${ }^{1}$ School of Transportation Science and Engineering, Harbin Institute of Technology, Harbin, China \\ ashenzhang@hit.edu.cn, 'ow.quanwei@163.com
}

Keywords: Freeway Traffic Conditions; Multiple Stakeholders; Data Envelopment Analysis.

\begin{abstract}
Effective assessment of traffic conditions is a key issue involved in alleviating freeway congestion, evaluating capital improvements and estimating travel time. Since the goals and objectives of assessment are inherently an expression of the various stakeholders affected by the traffic conditions, the assessment process and result must address the interests of all stakeholders. In this paper, a methodology and its application to assess traffic conditions on urban freeways are described. The methodology, which synthesizes Data Envelopment Analysis (DEA) and Analytical Hierarchy Process (AHP), can devise an overall traffic conditions assessment regarding various stakeholders' preferences. Application of the methodology to six real-life freeway corridors in Jilin Province indicated that the stakeholders can gain new insight into the overall traffic conditions behind multiple performance measures with our method, and the assessment results is helpful in identifying transportation investment priorities for specific regions and improving resource utilization among competing sectors.
\end{abstract}

\section{Introduction}

Understanding the temporal and spatial evolution of traffic conditions is a critical step toward improving freeway modeling and operations. With this clear understanding, administrators at any time can manage traffic congestion and incidents, Planners can determine whether congestion bottlenecks can be alleviated by improving operations or by minor capital improvements. Commuters can obtain the shortest route and travel time estimation to plan their trips. The widespread availability of freeway sensor data makes multivariate traffic condition analysis possible in ways that were not available in the past. However, there are two issues needed to be addressed during the process.

Firstly, the goals and objectives of traffic conditions assessment are inherently an expression of the various stakeholders affected by transportation system. This includes not only the administrators and the planners of transportation but also the commuters that experience their trips. In the principles of some multivariate statistical methods, all the stakeholders are treated as Decision Makers (DM), who have preferences to which of the measures they consider to be "strong important", "equal important" or "less important" measures. Performance assessment should allow decision makers to compare actual performance with desired performance as well as to provide the basis for making decisions to improve traffic conditions. Therefore, it is an urgent need to proactively involve various kinds of decision makers in the measurement process, and ensure the result to reflect their goals and objectives.

Secondly, identification of the need for and the effect of transportation infrastructure investment is particularly important in the assessment when development resources are scarce as in the case of developing countries or regions. From the perspective of public development agencies and planning authorities, investment of resources involves the identification and assessment of the need for infrastructure development as well as an accurate measurement of the need to allow for effective allocating of resources. For example, when the decision makers find that an increase in the road width can result in a more than proportional increase in the traffic volume and flow speed, they may intend to initiate development processes through proactive measures such as infrastructure investment, and 
vice versa. Therefore, the analysis of transportation investment needs and optimal allocation of resources seem to exhibit returns to scale, which is of considerable importance for the multivariate analysis of traffic conditions.

This paper introduces a novel hybrid method based on Data Envelopment Analysis (DEA) and Analytical Hierarchy Process (AHP). This method can assess traffic conditions of each freeway section relative to others by considering various stakeholders' preferences in multiple performance measures. In particular, this paper summarizes how the method uses surveillance data to assist in assessing freeway conditions, evaluating capital and operational improvements, it assesses the traffic conditions of 6 freeway sections in Jilin Province by incorporating two types of stakeholders' preferences, and multiple measures are established on the basis of the 12-month loop data for the year 2011. As a result, the best sections and others' performance gaps can be identified. The conclusions indicate the stakeholders can gain new insight into the overall traffic conditions behind multiple performance measures with our method, and the analysis of returns to scale can generate references for infrastructure investment and facilitate optimal allocation of resources It should be noted that this study is not intended as the definitive work on freeway capability measurement; instead, it describes our experience in learning more about freeway conditions assessment.

This paper is outlined as follows. Section 2 discusses the state of the art. Section 3 presents the hybrid evaluation model based on DEA and AHP, then details fundamental strategy of incorporating Decision-Making preference and estimating returns to scale. To verify the proposed method, an experiment is demonstrated and its results are analyzed in Section 4. Section 5 closes with a conclusion.

\section{Related Work}

In recent years, demands for a better understanding of the traffic conditions have stimulated a considerable research interest. Choe and Chen et al. developed a freeway performance measurement system (PeMS) for all of California [1], while Bertini et al. described the evolution of traffic conditions and measured bottleneck outflows by using this system [2]. Although PeMS can assist users compute some basic performance measures by processing real-time detector data, it hasn't devised an overall traffic conditions evaluation based on the multiple performance measures. Zuduo et al. analyzing important features related to bottleneck activations by Using wavelet transform, WT method can effectively identify the location of an active bottleneck, but lacks the capability to identify the relative congested or clear corridors [3]. Kamarianakis et al. forecasted the traffic flow conditions in an urban network based on data sets from loop detectors [4]. They concentrated on statistical methods, such as ARIMA, VARMA, and STARIMA models, but took the relative velocity as the only measure for traffic conditions. Hussein et al. demonstrated the feasibility of developing data fusion neural network architectures for incident risk detection on urban arterials using data from loop detector and probe vehicles [5]. However, the $\mathrm{NN}$ applications in transportation do not target interpretation of effects and signs, but rather aim at providing predictions for the phenomenon [6]. Turochy et al. measured variability in traffic conditions by applying principles of MSQC (multivariate statistical quality control) to derive a variability index [7]. In their study, the variability index is computed by measuring the size (spatial volume), mean speed, and occupancy with the detector data. While Catbagan et al. analyzed and compared possible two-lane expressway performance measures and recommended the suitable measures that would best describe the traffic flow characteristics [8]. The measures stated above are also proposed in our study to evaluate the traffic conditions.

\section{Methodology}

In this section, we will briefly formulate and discuss our integrated evaluation method based on DEA and AHP, the steps in incorporation of stakeholders' preferences and estimation of returns to scale will be introduced in the following subsections as well. 


\subsection{Method Description}

Let us assume that there are $n$ freeway sections to be assessed, which can be written as: $S=\left(S_{1}\right.$, $\left.S_{2}, \ldots, S_{n}\right)$. Each section consumes varying amounts of $m$ different inputs to produce $s$ different outputs. The input measures can be any factors used as a resource by the freeway, for example, the time, capital investment and transportation infrastructure. The output measures are the vehicle volume, occupancy density, incidents or other outcomes accommodated or produced by the freeway.

We first establish the freeway conditions evaluation models by synthesizing the cone ratio DEA model $\left(\mathrm{C}^{2} \mathrm{WH}\right)$ (Charnes and Cooper, 1989) and AHP. Our hybrid assessment model can be expressed in linear program (LP) form Eq. 1 and dual form Eq. 2 as shown below.

$$
\begin{gathered}
\left(P_{\mathrm{C}^{2} \mathrm{WH}-\mathrm{AHP}}\right)=\left\{\begin{array}{c}
\theta_{u}=\max \left(\mu^{T} y_{u}\right) \\
\omega^{T} x_{j}-\mu^{T} y_{j} \geq 0, j=1, \ldots, n \\
\omega^{T} x_{u}=1 \\
\omega \in V_{A H P}, \mu \in U_{A H P}
\end{array}\right. \\
\left(D_{\mathrm{C}^{2} \mathrm{WH}-\mathrm{AHP}}\right)=\left\{\begin{array}{c}
\min \left(\theta_{u}\right) \\
\sum_{j=1}^{n} x_{j} \lambda_{j}-\theta x_{u} \in V^{*}{ }_{A H P} \\
-\sum_{j=1}^{n} y_{j} \lambda_{j}+y_{u} \in U^{*}{ }_{A H P} \\
\lambda_{j} \geq 0, j=1,2, \ldots, n
\end{array}\right.
\end{gathered}
$$

In our assessment model, the Decision-Making preferences of stakeholders are introduced in the form of $V_{A H P}, V_{A H P}^{*}, U_{A H P}$ and $U_{A H P}^{*}$ generated using AHP.

The scalar variable $\theta$ in Eq. 1 and Eq. 2 represents the nonnegative performance score of each freeway section, and it ranges from 0 to 1 . If $S_{u}\left(S_{u} \in S\right)$ receives the optimal value $\theta_{u}=1$, then it is of relative high performance, but if $\theta_{u}<1$, it is of relative low performance.

Furthermore, since the value of $\theta_{u}$ means that $S_{u}$ can still achieve a minimum decrease of $\theta_{u}$ times in its inputs without decreasing the production for any outputs, the performance of $S_{u}$ is relatively lower when the $\theta_{u}$ is relatively smaller.

Besides the performance score $\theta$ in Eq. 1 and Eq. 2, we also calculate another two variables: $\omega$ and $\mu$.

The $\omega=\left(\omega_{1}, \omega_{2}, \ldots, \omega_{m}\right)^{T}$ and $\mu=\left(\mu_{1}, \mu_{2}, \ldots, \mu_{m}\right)^{T}$ in (1) are the preference weights of input and output measures, so the values of $\omega$ and $\mu$ reflect the relative importance of the input and output measures in stakeholders' preferences.

\subsection{Preference Incorporation}

The hybrid assessment model can incorporate preference in order to bring the results closer to the prior perceptions of stakeholders. The preferences are aggregated into "input /output preference cones", which are in the form of $V_{A H P}$ and $U_{A H P}$ (see Eq. 1), while $V_{A H P}^{*}$ and $U^{*}{ }_{A H P}$ (see Eq. 2) are the negative polar cones of $V_{A H P}$ and $U_{A H P}$. The "input/output cone" reflects the relative importance of each input/output measure regarding various stakeholders' preferences.

The AHP method is utilized to provide a vector of preference weights expressing the relative importance for each traffic measure and construct the "preference cones". The scale of relative importance is defined according to Satty 1-9 scale for pairwise comparison (Satty,1990). According to group AHP theory (Lai and Wong, 2002), single stakeholder's judgment can also be aggregated into a group judgment. Therefore, we obtain a $\mathrm{m} \times \mathrm{m}$ matrix $A_{m}$ for input measures and a $\mathrm{s} \times \mathrm{S}$ matrix $B_{s}$ for output measures. $A_{m}$ and $B_{s}$ are called judgment matrix.

Then the input/output preference cones $U, U^{*}, V$ and $V^{*}$ can be constructed using the following Eq. 3 ( $E$ is identity matrix, $\lambda$ is the max eigenvalue):

$$
\begin{array}{lll}
A=A_{m}-\lambda_{A} E_{m} & V_{A H P}=\{\omega \mid A \omega \geq 0\} & V_{A H P}^{*}=\left\{A^{T} \omega \mid \omega \leq 0\right\} \\
B=B_{s}-\lambda_{B} E_{s} & U_{A H P}=\{\mu \mid B \mu \geq 0\} & U_{A H P}^{*}=\left\{B^{T} \mu \mid \mu \leq 0\right\}
\end{array}
$$


In the above formula, the AHP solution is used to provide a regional value of traffic stakeholders' preferences. Since the regional value actually represents the preference weight region for input and output measures, the DEA model weighted by AHP proposed in our study is adopted to identify the optimal weight vectors in this solution region, and then evaluate the relative traffic conditions regarding preferences of various stakeholders. Therefore, this synthesis method can lead to a reasonable evaluation result that considers both objective loop-data characteristic and subjective stakeholders' preferences.

\section{Experimental Design}

\subsection{Data}

To illustrate our approach, the traffic conditions are evaluated with respect of various preferences by using the monthly data from Jilin Highway Administration. The data is mainly derived from the loop detector network, taken at 20 -second intervals. Our experiment covers the period from January 1, 2010 to December 31, 2010 and is for up to 6 sections on urban freeway network, the 12-month data for one section is treated as 12 sets of data from 12 different pseudo freeway sections. This generates $72(12 \times 6)$ data sets to be evaluated across time and space. The 6 freeway study stations are $\mathrm{K} 41+145$, which is located on Yanji, southbound mainline; K78+175, which is located on Yitong, southbound mainline; K93+005, which is located on Dunhua, eastbound mainline; K126+695, which is located on Tonghua, southbound mainline; K150+85, which is located on Songyuan, eastbound mainline; and K184+65, which is located on Liaoyuan, eastbound mainline.

\subsection{Computed Measures}

A large catalogue of measures can be computed from a stream of 20-second observations of loop detectors network and other surveillance systems. Six promising indicators including observation time, Investment amount, lane number, station total volume, station total occupancy, and station total weighted speed, which can reflect traffic conditions in a clear way, are derived for the assessment process. Other potential measures, such as incidents and vehicle length, are not analyzed.

Among the 6 chosen measures, the observation time, investment amount and lane number are taken as the input performance measures, while the rest will be treated as the output performance measures used in our assessment models.

\subsection{Results and Analysis}

To discuss the merits of our method, we prepare the stakeholders' preferences regarding the six input and output measures stated above. To simplify our discussion, we only consider the impact of subjective preference on the three output factors. Further, suppose there are two types of stakeholders, administrators and commuters. Administrators need to make strategic decisions on transportation infrastructure improvement occasionally, so they may prefer traffic volumes and vehicle density to flow speed. When they have "strong important" perception on the former, a judgment matrix $B_{\text {admin }}$ can be calculated (see Section 3.2). On the other hand, commuters pay more attention to the shorter travel time and more comfortable experience, so they may prefer vehicle speed and density to volumes. When they have "strong important" perception on the former, another judgment matrix $B_{\text {commuter }}$ is established. The two matrices are given as follows:

$$
B_{\text {admin }}=\left[\begin{array}{ccc}
1 & 1 & 5 \\
1 & 1 & 5 \\
1 / 5 & 1 / 5 & 1
\end{array}\right] \quad B_{\text {commuter }}=\left[\begin{array}{ccc}
1 & 1 / 5 & 1 / 5 \\
5 & 1 & 1 \\
5 & 1 & 1
\end{array}\right]
$$

Then the output preference cones $U_{\text {admin }}$ and $U_{\text {commuter }}$ can be constructed based on the Eq. 3 . By incorporating these preference cones into the Eq. 1, the performance evaluation results of these 72 dataset regarding two types of preference are calculated and shown in Fig. 1 and Fig. 2. Fig. 1 displays the preference score incorporating $U_{\text {admin }}$, while Fig. 2 describes the score incorporating $U_{\text {commuter }}$. The 6 series represent separately evaluation results of the 6 selected roadway segments.

As stated in Section 3.1, the plotted points, which fall on the horizontal solid black line in Fig. 1 and Fig. 2, are identified as relatively high performance ones. While the other points, which fall below the horizontal black line, are all of relative low performance in these series, and the performance gaps 
can be further identified with reference to their values. As a result, the traffic conditions of February, March and June observed in K184+65, the conditions of February observed in K126+695, and the conditions of July observed in K78+175 are the best from administrator's perspective. While the conditions of February, July and September observed in K126+695 are the best from commuter's perspective.

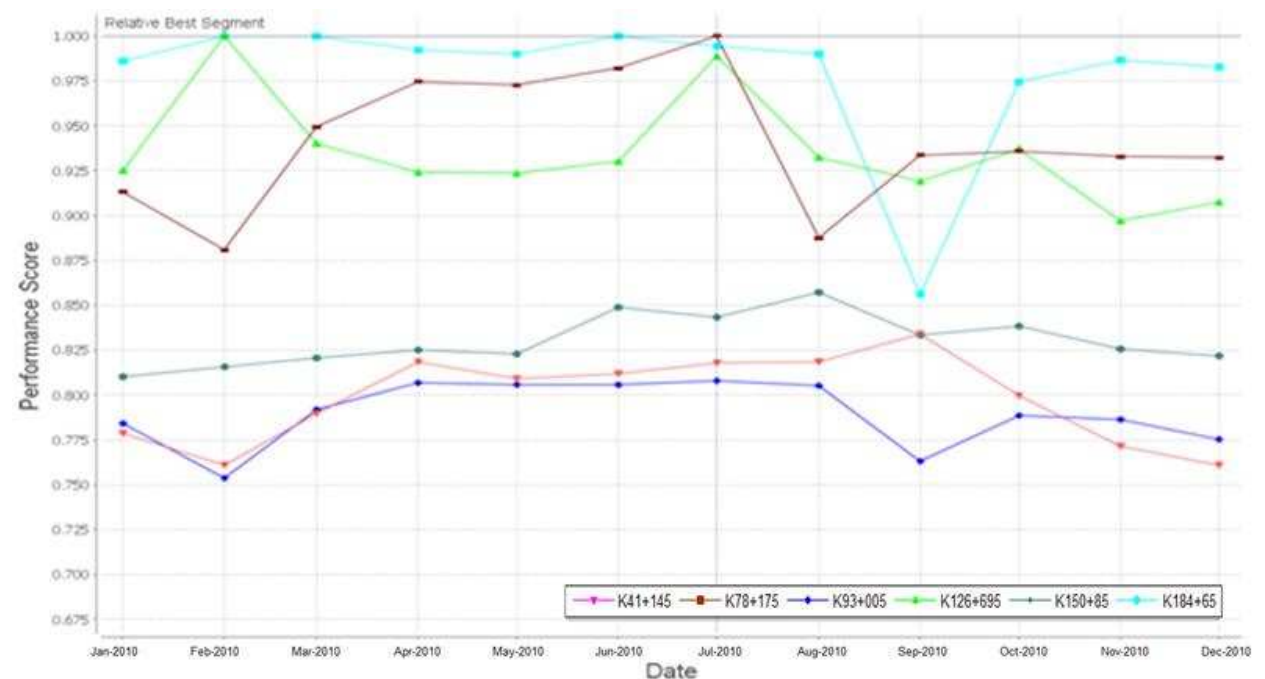

Figure 1 Performance score distribution with preferences of administrators

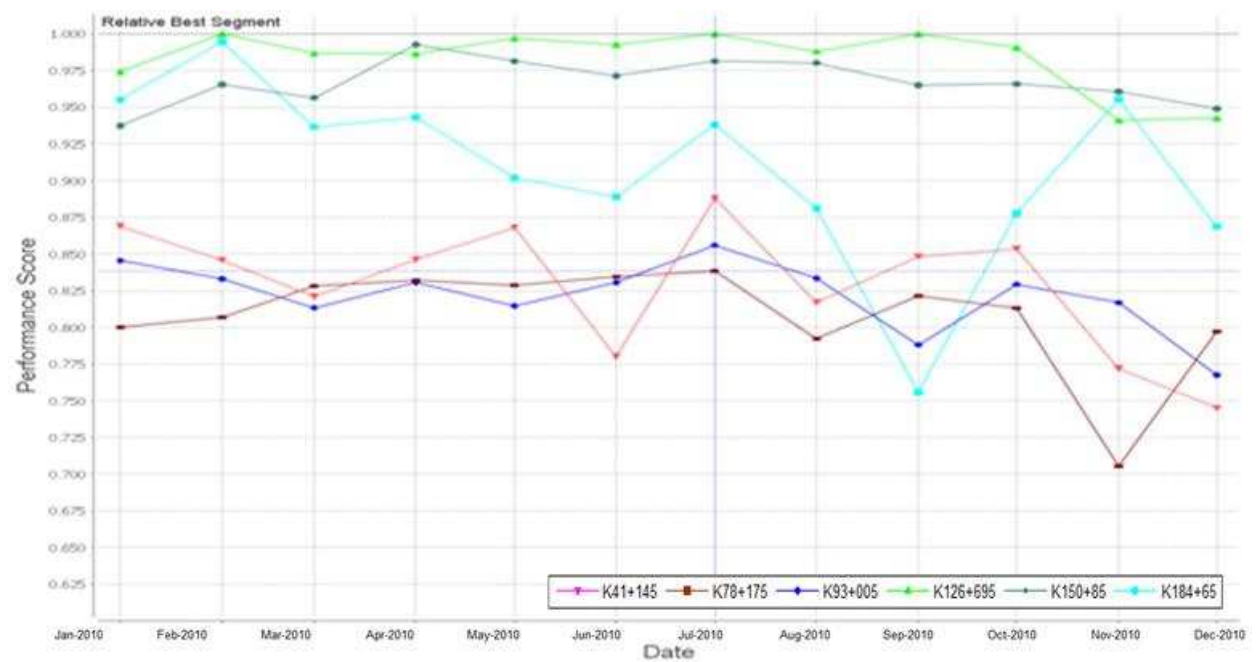

Figure 2 Performance score distribution with preferences of commuters

However, from the above figures, it can be observed that the same freeway segment's evaluation results may sometimes vary significantly across months. For example, the brown series in Fig. 1, which represents the K $78+175$, has a best performance score $\theta=1$ at July, but surprisingly, it has a steeply falling at August and its score $\theta$ is just 0.887554 . The average volume per minute $Q / T$ and the average occupancy $O / T$ of July is $(58.358,8.36 \%)$, while these measures of August is $(51.151$, $8.582 \%)$. The difference of the two-month measures causes the performance variation. There is still two issues should explained. Firstly, the reason, why the comparison of volume $(58.358>51.151)$ leads to an undesirable result - occupancy $(8.36 \%<8.582 \%)$, is that the weighted average speeds $V / Q$ of July and August are $(58.99>55.74)$, so the higher speed results in a lower vehicle density. Secondly, the likely reason for the large variation in volume is that July is a month of atypical traffic flow characteristics because most of the region population takes vacation at that time, whereas August is considered a typical one. As expected, the volumes of Yitong southbound mainline are separately 58.358 and 51.151 in July and August.

Besides, according to the comparison of Fig. 1 and Fig. 2, we find that the same dataset' score $\theta$ with different preferences may also differ widely from each other. Once again, take K $78+175$ for example, the brown series has the best $\operatorname{score} \theta=1$ at July as shown in Fig. 1, but in Fig. 2 its capability 
score $\theta$ is just around 0.6 when evaluation process incorporates commuters' preference $U_{\text {commuter }}$. The reason for this difference can be attributed to the impact of Decision-Making preferences. To help clarify these issues, we adopt Eq. 1 in (see Section 3.1) to calculate the preference weights of output metrics $(\mu)$ and explain the discrepancy. The weights vector $\mu$ for output metrics under different restrictions are shown below.

When with administrators' preferences $U_{\text {admin }}$ :

$$
S_{\text {July }}: \theta_{a}=1.000 \mu=\left(\mu_{\text {volume }}, \mu_{\text {occupancy }}, \mu_{\text {speed }}\right)^{T}=(4.22,4.42,1.28)^{T}
$$

When with commuters' preferences $U_{\text {commuter }}$.

$$
S_{\text {July }}: \theta_{c}=0.838 \mu=\left(\mu_{\text {volume }}, \mu_{\text {occupancy }}, \mu_{\text {speed }}\right)^{T}=(1.25,4.09,4.54)^{T}
$$

It is obvious that the distinct output weights have imposed preference restrictions for the output measures. While the evaluation process adopts the preference cone $U_{\text {admin }}$. "volume" and "occupancy" have the greatest impact on the final results. Since $S_{\text {july }}$ of K $78+175$ holds a very high value for $Q$ against the other 11-month datasets in Fig. 1. the performance scores of the other 11 datasets generally appear with relative low values. However, when the evaluation process incorporates $U_{\text {commuter }}$, the measures of "speed" and "occupancy" have the greatest weights. Because all the datasets of $\mathrm{K} 78+175$ have nearly the same value for $V$ except the dataset from November, the brown series has a relative smooth curve in Fig. 2, except for the eleventh point. This can explain the reason why $S_{j u l y}$ of K $78+175$ varies significantly with different output preference cones.

\section{Acknowledgements}

This research is supported by the State Key Program of National Natural Science of China (Grant No. 51138003), and the Fundamental Research Funds for the Central Universities (Grant No. HIT.NSRIF.201184).

\section{References}

[1] T. Choe, A. Skabardonis, and P. Varaiya. Freeway Performance Measurement System: Operational Analysis Tool. In Transportation Research Record: Journal of the Transportation Research Board, No. 1811, Transportation Research Board, Washington, D.C., (2002) 67-75.

[2] R. L. Bertini, and A. M. Myton. Use of Performance Measurement System Data to Diagnose Freeway Bottleneck Locations Empirically in Orange County, California. In Transportation Research Record: Journal of the Transportation Research Board, No. 1925, Transportation Research Board, Washington, D.C., (2005) 48-57.

[3] Z. Zuduo, A. Soyoung, C. Danjue and L. Jorge. Applications of wavelet transform for analysis of freeway traffic: Bottlenecks, transient traffic, and traffic oscillations. Transportation Research Part B, 45, (2011) 372-384.

[4] Y. Kamarianakis, and P. Prastacos. Forecasting Traffic Flow Conditions in an Urban Network: Comparison of Multivariate and Univariate Approaches. In Transportation Research Record: Journal of the Transportation Research Board, No. 1857, Transportation Research Board, Washington, D.C., (2005) 74-84.

[5] D. Hussein and T. Kim. Development and evaluation of arterial incident detection models using fusion of simulated probe vehicle and loop detector data. Information Fusion, 12, (2011) 20-27.

[6] M.G. Karlaftis and E.I.Vlahogianni. Statistical methods versus neural networks in transportation research: Differences, similarities and some insights. Transportation Research Part C, 19, (2011) 387-399.

[7] R. E. Turochy, and B. L. Smith. Measuring Variability in Traffic Conditions by Using Archived Traffic Data. In Transportation Research Record: Journal of the Transportation Research Board, No. 1804, Transportation Research Board, Washington, D.C., (2002) 168-172.

[8] J. L. Catbagan, and H. Nakamura. Evaluation of Performance Measures for Two-Lane Expressways in Japan. In Transportation Research Record: Journal of the Transportation Research Board, No. 1988, Transportation Research Board, Washington, D.C., (2006) 111-118. 